University of Nebraska - Lincoln

DigitalCommons@University of Nebraska - Lincoln

Faculty Publications from the Department of Engineering Mechanics

Mechanical \& Materials Engineering,

Department of

6-25-2004

\title{
Spinning Continuous Fibers for Nanotechnology
}

Yuris A. Dzenis

University of Nebraska-Lincoln, ydzenis@unl.edu

Follow this and additional works at: https://digitalcommons.unl.edu/engineeringmechanicsfacpub

Part of the Mechanical Engineering Commons

Dzenis, Yuris A., "Spinning Continuous Fibers for Nanotechnology" (2004). Faculty Publications from the Department of Engineering Mechanics. 50.

https://digitalcommons.unl.edu/engineeringmechanicsfacpub/50

This Article is brought to you for free and open access by the Mechanical \& Materials Engineering, Department of at DigitalCommons@University of Nebraska - Lincoln. It has been accepted for inclusion in Faculty Publications from the Department of Engineering Mechanics by an authorized administrator of DigitalCommons@University of Nebraska - Lincoln. 


\title{
Spinning Continuous Fibers for Nanotechnology
}

\author{
Yuris Dzenis \\ The author is in the Department of Engineering Mechanics, University of Nebraska-Lincoln, \\ Lincoln, NE 68588-0526, USA; email:ydzenis@unl.edu
}

$\mathbf{N}$ anotubes of carbon and other materials are arguably the most fascinating materials playing an important role in nanotechnology today. Their unique mechanical, electronic, and other properties are expected to result in revolutionary new materials and devices. However, these nanomaterials, produced mostly by synthetic bottomup methods, are discontinuous objects, and this leads to difficulties with their alignment, assembly, and processing into applications. Partly because of this, and despite considerable effort, a viable carbon nanotube-reinforced supernanocomposite is yet to be demonstrated. Advanced continuous fibers produced a revolution in the field of structural materials and composites in the last few decades as a result of their high strength, stiffness, and continuity, which, in turn, meant processing and alignment that were economically feasible. Fiber mechanical properties are known to substantially improve with a decrease in the fiber diameter. Hence, there is a considerable interest in the development of advanced continuous fibers with nanoscale diameters. However, conventional mechanical fiber spinning techniques cannot produce fibers with diameters smaller than about $2 \mu \mathrm{m}$ robustly. Most commercial fibers are several times that diameter, owing to the trade-offs between the technological and economic factors.

Electrospinning technology enables production of continuous polymer nanofibers from polymer solutions or melts in high electric fields. When the electric force on induced charges on the polymer liquid overcomes surface tension, a thin polymer jet is ejected. The charged jet is elongated and accelerated by the electric field, undergoes a variety of instabilities, dries, and is deposited on a substrate as a random nanofiber mat. The first patent on the process was awarded in 1934; however, outside of the filter industry, there was little interest in the electrospinning or electrospun nanofibers, until the mid-1990s (1). Since that time, the process attracted rapidly growing interest triggered by potential applications of nanofibers in the nanotechnology. The publication rate has nearly doubled annually, reaching about 200 articles in 2003. Over a hundred synthetic and natural polymers were electrospun into fibers with diameters ranging from a few nanometers to micrometers (see the figure, panel A).

The main advantage of this topdown nanomanufacturing process is its relatively low cost compared to that of most bottom-up methods. The resulting nanofiber samples are often uniform and do not require expensive purification (panels B and C). Unlike submicrometer-diameter whiskers, inorganic nanorods, carbon nanotubes, and nanowires, the electrospun nanofibers are continuous. As a result, this process has unique potential for costeffective electromechanical control of fiber placement and integrated manufacturing of two- and three-dimensional nanofiber assemblies. In addition, the nanofiber continuity may alleviate, at least in part, concerns about the properties of small particles (2). Nanofibers are expected to possess high axial strength combined with extreme flexibility. The nanofiber assemblies may feature very high open porosity coupled with remarkable specific surface area. Yet, these assemblies would possess excellent structural mechanical properties. Uses of nanofibers in composites, protective clothing, catalysis, electronics, biomedicine (including tissue engineering, implants, membranes, and drug delivery), filtra- tion, agriculture, and other areas are presently being developed. Clearly, there is a growing interest in the process, but the results reported to date are centered mostly on the empirical production and the proposed uses of polymer nanofibers. At the same time, thorough understanding of the mechanisms of jet formation and motion is needed for the development of robust methods of process control. Analysis of the electrospinning process is complicated by electromechanical coupling, nonlinear rheology, and unusual jet instabilities. Some progress was recently made on modeling of jet initiation (3, 4). Steady-state spinning was modeled in the nonlinear rheologic regime important for polymer jets $(5,6)$. Experimental observations and modeling of bending (or whipping) instability (7, 8) produced a major breakthrough in process analysis. It substantially improved our understanding of the jet motion and removed an early controversy in the electrospinning studies over the interpretation of long-exposure time images of the instability process zone $(1,7,8)$. It has been suggested $(7,8)$ that bending instabilities constitute a major mechanism responsible for the rapid jet thinning in this process. These instabilities are also responsible for the resulting random nanofiber orientation.

More recently, three major breakthroughs were made that are expected to have lasting impact on the quality and scope of the applications. First, several methods of nanofiber alignment were developed that can be roughly classified into methods "directing" (9-12) or suppressing $(13,14)$ jet bending instabilities. The methods need to be further improved because most produce only partial alignment, but the results show promise. Align- 

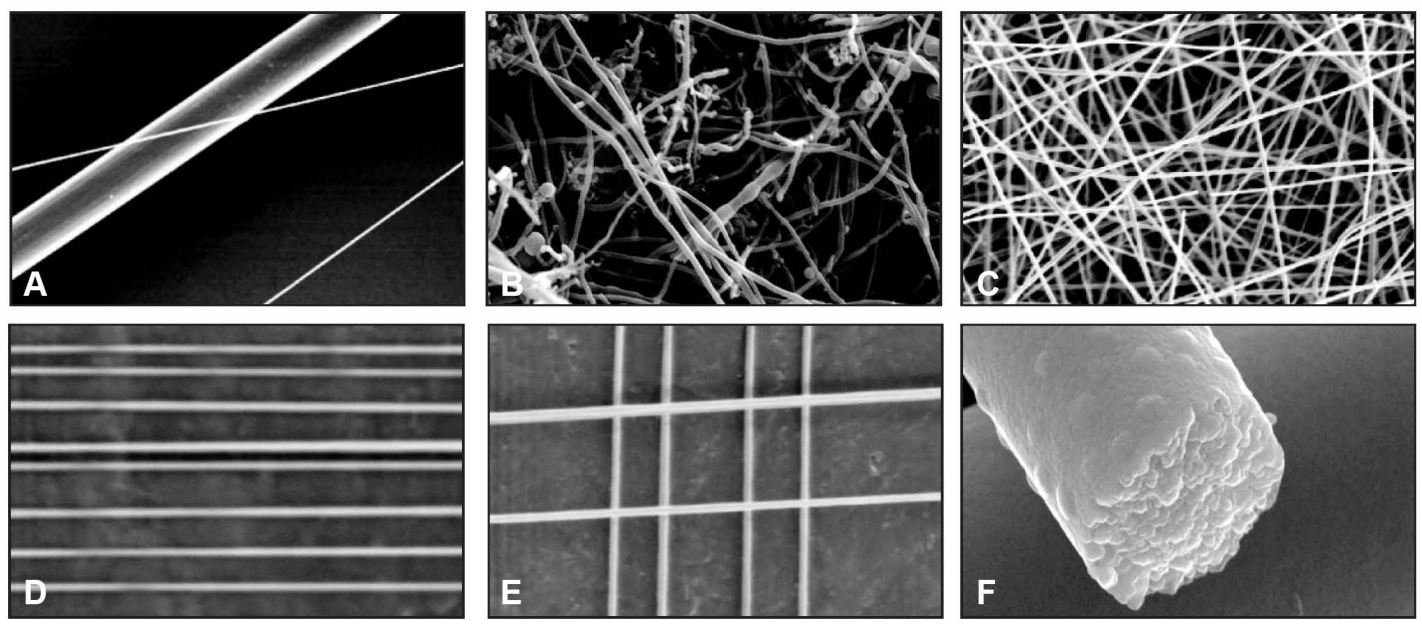

Figure: The next nanotech? (A) Comparison of commercial advanced carbon fiber, one of the smallest advanced fibers available, and electrospun continuous nanofiber. Comparison of (B) vapor-grown commercial carbon nanofibers and (C) electrospun carbon nanofibers showing substantially better nanofiber uniformity and sample purity. (D and E) Examples of highly aligned and spaced linear and orthogonal assemblies of continuous nanofibers produced by the gap method of alignment (I2). (F) Cross section of continuous nanocrystalline zirconia nanofiber for potential applications in supertough ceramics.

ment can revolutionize existing applications of nanofibers and help develop entirely new ones. The modified methods demonstrate the feasibility of integrated nanofiber manufacturing and placement or assembly (see the figure, panels $\mathrm{D}$ and $\mathrm{E}$ ) that can be extremely economic when compared to the post-processing methods that are currently being developed for carbon nanotubes.

In the second breakthrough, the original process used with high molecular weight polymers has been modified and applied, in combination with sol-gel chemistry, to produce continuous ceramic nanofibers (15-18). These nanofibers can be beneficial in the areas of catalysis, tough and high-temperature ceramics, active and sensing materials, and many others. Nanocrystalline nanofibers such as the one shown in panel $F$ of the figure may lead to supertough ceramics by simultaneous use of the two known methods of toughening - that is, fiber reinforcement and nanocrystallinity. The generality of the sol-gel technique opens broad opportunities to produce and use novel inorganic and hybrid nanofibers from a variety of materials. Along with the polymer and polymer-derived carbon and ceramic nanofibers, the sol-gel-derived ceramic nanofibers provide a comprehensive nanomaterial and nanomanufacturing platform for an extremely broad variety of applications.
In the third recent breakthrough, a method of coaxial electrospinning was developed and used to produce continuous coated and hollow nanofibers (19-21). This method allows a singlestep production of continuous nanotubes or nanopipes that complements the multistep template methods of tube production demonstrated earlier.

Despite considerable recent progress on the process modification and control, serious challenges remain. Improved models of the process are needed to achieve better understanding of the mechanisms of jet thinning. In particular, thermal and mass transport within the jet in conjunction with solvent evaporation seem to be crucial for jet thinning, solidification, and formation of nanofiber molecular structure. The evaporation from the thin jets in electrospinning is believed to be rapid. Yet, it is expected to lead to inhomogeneous transient concentration and temperature profiles that will substantially affect the local rheological and other properties of the fluid. Only rudimentary attempts to incorporate the solvent evaporation in the electrospinning analysis have been made so far (7). When developed, a more rigorous model can provide insights into jet thinning as well as into the mechanisms and conditions for other interesting observed phenomena such as formation of pores during the electrospinning with highly volatile solvents, formation of ribbonlike fiber shapes, and others (22-25).
The major experimental challenge is to develop robust methods for manufacturing extremely small nanofibers. Although diameters as small as 3 to 5 nm were reported $(26,27)$, nanofibers smaller than about $50 \mathrm{~nm}$ in diameter cannot currently be produced uniformly and repeatedly for most materials systems. The analysis of the effects of solvent evaporation described above may prove useful for this purpose. Also, the effects of instabilities on the diameter reduction need to be studied more carefully. According to $(7,8)$, the primary bending or whipping instability has a major effect on the reduction of the jet diameter. However, this contradicts experimental data reported in $(13,14)$ where this instability was suppressed by two different methods (secondary field and short spinning distance), yet the modified processes resulted in nanofiber diameters comparable to the diameters in the conventional process with instabilities. Thorough experimental analysis of the composition of the electrospinning instability zone may provide insight into the temporal and spatial evolution of jet instabilities and jet thinning.

Another need is to model nanofiber deposition on substrates, both stationary and moving, which can help improve the nanofiber alignment using the newly developed methods and may also lead to the development of novel techniques. Multiple jet electrospinning should also be analyzed, 
and jet interactions with each other as well as with the modified field configurations used in the alignment methods should be characterized and modeled. Multiple jets are critical for the scale-up of the nanofiber production process. New elegant processes yielding high aerial jet densities, similar to the multiple jet spinning method reported in (28), are expected to be developed. Analysis and implementation of the combined methods involving ultrasmall-diameter jets, alignment, and multiple jet spinning are expected to be especially challenging.

Electrospun continuous polymer, carbon, and ceramic nanofibers have considerable advantages when compared to the discontinuous carbon or other nanotubes or nanorods in terms of the cost, health concerns, and the possibility of integrated one-step manufacturing of assemblies. A fundamental experimental and theoretical analysis of the process is needed to develop flexible and reliable methods of fabrication of nanofibers and their assemblies and composites.

\section{References and Notes}

I. D. H. Reneker, I. Chun, Nanotechnology 7, 216 (1996).

2. "For science, nanotech poses big problems," Washington Post, 3I January 2004.

3. A. F. Spivak, Y.A. Dzenis, J. Appl. Mech. 66, 1026 (I999).

4. A. L. Yarin, S. Koombhongse, D. H. Reneker, J. Appl. Phys. 90, 4836 (200I).

5. A. F. Spivak, Y. A. Dzenis, D. H. Reneker, Mech. Res. Commun. 27, 37 (2000).

6. J. J. Feng, J. Non-Newtonian Fluid Mech. I I6, 55 (2003).

7. A. L. Yarin, S. Koombhongse, D. H. Reneker, J. Appl. Phys. 89, 3018 (200I).

8. Y. M. Shin et al., Appl. Phys. Lett. 78, II 49 (200I).

9. A. Theron, E. Zussman, A. L.Yarin, Nanotechnology I 2, 384 (200I).

I0. R. Dersch et al., J. Polym. Sci. A Polym. Chem. 4I, 545 (2003).

II. D. Li, Y.Wang, Y. Xia, Nano Lett. 3, II 67 (2003).

12. An alignment method similar to the one described by Dersch et al. (I0) and Li et al. (II) has been used in my laboratory since 2001 .

13. J. M. Deitzel et al., Polymer 42, 8163 (200I).

14. J. Kameoka, H. G. Craighead, Appl. Phys. Lett. 83, 37I (2003).

I5. Y. A. Dzenis, G. Larsen, U.S. patent pending (200I).
16. G. Larsen et al., J. Am. Chem. Soc. I 25, I I54 (2003).

17. H. Dai et al., Nanotechnology 13, 674 (2002).

18. S.-S. Choi et al., J. Mater. Sci. Lett. 22, 89 I (2003).

19. Z. Sun et al., Adv. Mater. I5, 1929 (2003).

20. I. G. Loscertales et al., J.Am. Chem. Soc. I 26, 5376 (2004).

2I. D. Li,Y. Xia, Nano Lett. 4, 933 (2004).

22. M. Bognitzki et al., Adv. Mater. I3, 70 (200I).

23. S. Megelski et al., Macromolecules 35, 8456 (2002).

24. L. Huang et al., Macromolecules 33, 2989 (2000).

25. S. Koombhongse,W. Liu, D. H. Reneker, J. Polym. Sci. B Polym. Phys. 39, 2598 (200 I).

26.Y. Zhou et al., Appl. Phys. Lett. 83, 3800 (2003).

27. H. Fong, D. H. Reneker, J. Polym. Sci. B Polym. Phys. 37, 3488 (1999).

28. A. L. Yarin, E. Zussman, Polymer 45, 2977 (2004).

29. I thank Y. Wen for providing the scanning electron microscope images. This article resulted from research supported by multiple agencies. The National Science Foundation provided support for electrospinning process analysis and ceramic nanomanufacturing. 